\title{
AVALIAÇÃO DA EFICÁCIA BIOLÓGICA DE HERBICIDAS APLICADOS EM PRÉ-EMERGÊNCIA PARA O CONTROLE DE PLANTAS DANINHAS NA CULTURA DA BATATA (Solanum tuberosum L.)'
}

\author{
JULIO PEDRO LACA-BUENDIA * * \\ JOSÉ FRANCISCO RABELO LARA ***
}

\begin{abstract}
Avaliou-se a performance dos herbicidas Corsum (Metolachlor - $840 \mathrm{~g} / \mathrm{L}+$ Metribuzin - $120 \mathrm{~g} / \mathrm{L}$ ), e Dual (Metolachlor $960 \mathrm{~g} / \mathrm{L}$ ) aplicados na préemergência para o controle de plantas daninhas, na cultura da batata, cultivar 'Achat', em solo Latossolo Vermelho, textura Franco-argilosa. Os tratamentos foram: Corsum a $240+1680 ; 300+2100$ e $360+2520$ g/ha; Metolachlor a 1920, 2400 e 2880 g/ha, testemunha capinada e sem capina. Conclui-se que Corsum e Metolachlor, nas doses testadas, demostraram $100 \%$ de eficácia no controle de Galinsoga parviflora e Brachiaria plantaginea, até 45 dias depois de aplicação. A competição com plantas daninhas apresentou redução na produção de batata. Após 60 dias da aplicação não foram descobertas diferenças entre os herbicidas nas doses testadas e a testemunha capinada, quanto ao número de tubérculos e diâmetro do tubérculo. Estes herbicidas são altamente seletivos, apresentando nota EWRC de 1,0 até 15 dias após aplicação.
\end{abstract}

\section{INTRODUÇÃO}

A batata constitui-se em excelente fonte de nutrientes para a população, segundo WOOLFE (1987) que constatou o alto valor biológico de sua proteina.

No Brasil, a produção média, no periodo de $1986-1990$ foi de 2,14 milhões de toneladas de batatas, cultivadas em 163.881 hectares, com produtividade média de 13 t/ha (CAMARGO FILHO et al., 1993).

- Trabalho apresentado no XXI Congresso Brasileiro da Ciência das Plantas Daninhas, Caxambu, Minas Gerais.

- Engenheiro Agrônomo, M.Sc. Pesq./Empresa de Pesquisa Agropecuária de Minas Gerais (EPAMIG), Belo Horizonte, MG.

** Biólogo, Pesq./EPAMIG, Prudente de Morais, MG. 
O Estado de Minas Gerais é o segundo maior produtor do pais, tendo alcançado, em 1993, a marca de 608.446 t. A região Sul de Minas destaca-se como maior produtora, representando $76 \%$ do total da produção do Estado (MASCARENHAS et al., 1995).

O periodo de controle das plantas daninhas que atacam esta cultura é de 56 dias (SAGHIR; MARKOUILIS, 1974)

Indicações de herbicidas para a cultura da batata, visando o controle das plantas daninhas, vêm sendo estudadas por vários pesquisadores, conforme pode ser observado nos trabalhos desenvolvidos por: SANTOS \& ROZANSKI (1980), SILVA \& CARMO (1980), CARDOSO (1981), PARRAGA et al. (1988), SOSA et al. (1988), MASCARENHAS et al. (1995) e DARIO et al. (1995).

O presente trabalho de campo teve por objetivo avaliar a performance dos herbicidas Corsum (Metolachlor $840 \mathrm{~g} / \mathrm{L}+$ Metribuzin 120 $\mathrm{g} / \mathrm{L}$ ) e Dual (Metolachlor $960 \mathrm{~g} / \mathrm{L}$ ), na aplicação de pré-emergência, para controle de plantas daninhas na cultura da batata.

\section{MATERIAL E MÉTODOS}

O ensaio de campo foi instalado na Fazenda Varginha, de propriedade do Sr. Milton Alves da Costa, no municipio de Sete Lagoas (MG), no inverno de 1995, em solo de Cerrado do tipo LatossoloVermelho, textura Franco-Argilo-Siltosa, com 3,02\% de matéria orgânica, $53 \%$ de argila, $33 \%$ de silte e $\mathrm{pH} 6,8$.

A cultivar utilizada foi a 'Achat', plantada em 19/08/95, utilizando-se o espaçamento de $0,80 \mathrm{~m} \times 0,35 \mathrm{~m}$ entre as plantas, com semeadura manual e densidade de dez plantas em cada parcela.

A adubação de plantio usada foi de $1500 \mathrm{~kg} / \mathrm{ha}$ da fórmula 4:14:8 de NPK. Após 35 dias da emergência foi realizada adubação nitrogenada de cobertura com $300 \mathrm{~kg}$ /ha de sulfato de amônia.

$\mathrm{O}$ ensaio foi instalado no desenho experimental de blocos casualizados, com quatro repetições em parcelas e quatro fileiras de $4,0 \mathrm{~m}$ de comprimento, espaçadas entre si em $0,80 \mathrm{~m}$, resultando em área total de $12,8 \mathrm{~m}^{2}$. Foram colhidas duas fileiras em cada parcela, representando área útil de $6,4 \mathrm{~m}^{2}$ (GOMES, 1973).

Os herbicidas foram aplicados em uma única vez na pré-emergência (Tabela 1), em 19/08/95, utilizando-se pulverizador costal pressurizado a $\mathrm{CO}_{2}$, dotado de barra com quatro bicos, do tipo leque 80.04 , a $0,5 \mathrm{~m}$ do solo, com vazão de $350 \mathrm{~L} / \mathrm{h}$, usando-se pressão constante de $40 \mathrm{lb} / \mathrm{pol} 2$, entre $16 \mathrm{~h}$ e $30 \mathrm{~min}$ e $18 \mathrm{~h}$. O tempo apresentava-se sem vento, o céu nublado e o solo úmido, após irrigação (infiltração por sulco), com temperatura do ar de $20,2{ }^{\circ} \mathrm{C}$ e umidade relativa de $34 \%$. 
Foram realizadas duas capinas manuais nas parcelas das testemunhas capinadas para o controle das plantas daninhas, não havendo nenhuma aplicação de pesticidas.

Utilizou-se método de irrigação de infiltração por sulco, com bordos fechados entre as fileiras das plantas, realizado duas vezes por semana, desde o plantio até 20 dias antes da colheita (DAKER, 1970).

TABELA 1 - TRATAMENTOS ESTUDADOS

\begin{tabular}{|c|c|c|c|}
\hline \multirow[t]{2}{*}{ Tratamentos } & \multicolumn{2}{|c|}{ Doses } & \multirow{2}{*}{$\begin{array}{l}\text { Modo de } \\
\text { Aplicação }\end{array}$} \\
\hline & (g/ha do i. a) & (L/ha do p. c.) & \\
\hline $\begin{array}{l}\text { 1-Corsum (Metolachlor }-840 \mathrm{~g} / \mathrm{L}+ \\
\text { Metribuzin }-120 \mathrm{~g} / \mathrm{L} \text { ) }\end{array}$ & $(240+1680)$ & 2,0 & Pré-emergência \\
\hline 2-Corsum & $(300+2100)$ & 2,5 & Pré-emergência \\
\hline 3-Corsum & $(360+2520)$ & 3,0 & Pré-emergência \\
\hline 4-Dual - $960 \mathrm{~g} / \mathrm{L}$ & 1920 & 2,0 & Pré-emergência \\
\hline 5-Dual & 2400 & 2,5 & Pré-emergência \\
\hline 6-Dual & 2880 & 3,0 & Pré-emergência \\
\hline 7-Testemunha capinada & -.- & -- & \\
\hline 8-Testemunha sem capina & $-\cdots$ & $\cdots$ & \\
\hline
\end{tabular}

i. $\mathrm{a}=$ Ingrediente ativo

$\mathrm{p.c}=$ Produto comercial

Avaliações visuais de fitointoxicação das plantas da batata foram realizadas aos 15 e 30 dias após aplicação dos herbicidas, mediante escala visual da European Weed Research Council (EWRC, 1964) com 9 pontos, em que 1 corresponde a nenhuma injúria e 9 significa morte da planta.

Aos 15,30 e 45 dias após a aplicação realizaram-se avaliações da eficácia de controle das plantas daninhas, utilizando-se escala percentual de 0 a $100 \%$, em que $0 \%$ corresponde a nenhum controle e $100 \%$ ao controle total (excelente), para cada espécie encontrada nas parcelas dos tratamentos. Na testemunha sem capina realizou-se contagem por espécie, dentro da área útil de cada parcela em $1,0 \mathrm{~m}^{2}$.

Quando as plantas atingiram a maturação em 08/11/95 realizou-se a dos tubérculos. Para determinação da altura média das plantas foram tomadas dez plantas ao acaso por parcela, aos 60 dias após a emergência. Contou-se o número total de tubérculos por parcela, bem como o número de tubérculos com diâmetro menor que $4,5 \mathrm{~cm}$ e seus respectivos pesos por parcela. 
Os dados foram submetidos à analise de variância, sendo as médias dos tratamentos comparadas pelo teste de Tukey ao nivel de $5 \%$ de probabilidade (GOMES, 1973).

\section{RESULTADOS E DISCUSSÃO}

As condições locais mostraram-se ótimas tanto para o desenvolvimento das plantas daninhas, como para a cultura da batata.

As espécies de plantas daninhas encontradas na área foram: Galinsoga parvifiora (fazendeiro), 43\%; Euphorbia heterophylla (leitero), $32 \%$; Brachiaria plantaginea (capim-marmelada), $20 \%$ e Bidens pilosa (picão-preto), $5 \%$.

De acordo com os resultados obtidos verificou-se que os herbicidas, nas doses estudadas, apresentaram nota $1(0 \%=$ nenhuma injúria) aos 15 e 30 dias após a aplicação, constatando-se alta seletividade para a cultura da batata.

Houve diferença significativa na produção dos tubérculos colhidos, sendo os melhores resultados alcançados quando aplicou-se Metolachlor a $2.880 \mathrm{~g} / \mathrm{ha}$ do ingrediente ativo (i.a.) e Metolachlor + Metribuzin a $360+2.520 \mathrm{~g} / \mathrm{ha}$ do i.a. (produção respectiva de $7.422 \mathrm{~kg} / \mathrm{ha}$ e 7.266 $\mathrm{kg} / \mathrm{ha}$ ). A testemunha capinada apresentou rendimento de $7.578 \mathrm{~kg} / \mathrm{ha}$ (Tabela 2).

TABELA 2 - RESULTADOS MÉDIOS OBTIDOS NA AVALIAÇÃO DA EFICÁCIA BIOLÓGICA DE HERBICIDAS APLICADOS EM PRÉEMERGÊNCIA, PARA O CONTROLE DE PLANTAS DANINHAS NA CULTURA DA BATATA - SETE LAGOAS (MG) - 1995

\begin{tabular}{|c|c|c|c|c|}
\hline Tratamentos & $\begin{array}{c}\text { Doses } \\
\text { (g/ha do i.a.) }\end{array}$ & $\begin{array}{c}\text { Produção } \\
(\mathrm{kg} / \mathrm{ha})\end{array}$ & $\begin{array}{c}\text { Altura de } \\
\text { Planta }{ }^{(1)}(\mathrm{cm})\end{array}$ & $\begin{array}{c}\text { Número total de } \\
\text { Tubérculos }\left(6,4 \mathrm{~m}^{2}\right)\end{array}$ \\
\hline Corsum $\left({ }^{*}\right)$ & $(240+1680)$ & $7.070 \mathrm{a}^{(2)}$ & $47,02 \mathrm{a}$ & $142,5 \mathrm{a}$ \\
\hline Corsum $\left({ }^{*}\right)$ & $(300+2100)$ & $6.992 \mathrm{a}$ & $47,05 \mathrm{a}$ & $149,5 \mathrm{a}$ \\
\hline Corsum $\left({ }^{*}\right)$ & $(360+2520)$ & $7.266 \mathrm{a}$ & $46,40 \mathrm{a}$ & $155,5 \mathrm{a}$ \\
\hline Dual & 1920 & $7.109 \mathrm{a}$ & $48,75 \mathrm{a}$ & $154,2 \mathrm{a}$ \\
\hline Dual & 2400 & $7.188 \mathrm{a}$ & $48,90 \mathrm{a}$ & $157,8 \mathrm{a}$ \\
\hline Dual & 2880 & $7.422 \mathrm{a}$ & $46,18 \mathrm{a}$ & $151,8 \mathrm{a}$ \\
\hline Testemunha capinada & $\cdots$ & $7.578 \mathrm{a}$ & $47,10 \mathrm{a}$ & $152,5 \mathrm{a}$ \\
\hline $\begin{array}{l}\text { Testemunha sem } \\
\text { capina }\end{array}$ & $\cdots$ & $703 \mathrm{~b}$ & $27,75 b$ & $23,5 b$ \\
\hline Média Geral & & 6.626 & 45,13 & 140,1 \\
\hline Valor de F & & $36,86(* *)$ & $20,97(* *)$ & $86,17(* *)$ \\
\hline DMS & & 1604 & 6,31 & 20,61 \\
\hline C.V. $(\%)$ & & 9,84 & 5,69 & 5,98 \\
\hline
\end{tabular}

(1) Tomada após 60 dias da emergência. (2) Médias na mesma coluna, seguidas pela mesma letra não diferem estatisticamente entre si, pelo teste de Tukey ao nivel de $5 \%$ de probabilidade.

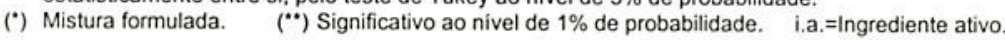


Verificou-se que a competição das plantas daninhas com a cultura apresentou efeito negativo na produção de tubérculos colhidos, sendo que a testemunha sem capina alcançou $703 \mathrm{~kg} / \mathrm{ha}$.

Para a altura da planta, número total de tubérculos por parcela, número e peso de tubérculos com diâmetro menor que $4,5 \mathrm{~cm}$ houve diferença significativa, sendo que nenhum dos herbicidas, nas doses estudadas, apresentaram diferença em relação a testemunha capinada (Tabela 3). TABELA 3 - RESULTADOS MÉDIOS OBTIDOS NA AVALIAÇÄO DA EFICÁCIA
BIOLÓGICA DE HERBICIDAS APLICADOS EM PRÉEMERGENCIA, PARA CONTROLE DE PLANTAS DANINHAS NA CULTURA DA BATATA - SETE LAGOAS (MG) - 1995

\begin{tabular}{|c|c|c|c|c|c|}
\hline \multirow[t]{2}{*}{ Tratamentos } & \multirow[t]{2}{*}{$\begin{array}{c}\text { Doses } \\
\text { (g/ha do i.a.) }\end{array}$} & \multirow{2}{*}{$\begin{array}{l}\text { Números } \\
\text { Tubérculos } \\
\text { Diâmetro } \\
<4,5 \mathrm{~cm}\end{array}$} & \multirow{2}{*}{$\begin{array}{c}\text { Peso de } \\
\text { Tubérculos } \\
\text { Diâmetro } \\
>4,5 \mathrm{~cm}\end{array}$} & \multicolumn{2}{|c|}{$\begin{array}{l}\text { Fitointoxicação } \\
\text { (EWRC } 1 \text { a 9) }\end{array}$} \\
\hline & & & & $15 \mathrm{DAT}$ & $30 \mathrm{DAT}$ \\
\hline Corsum $(*)$ & $(240+1680)$ & $140,5 \mathrm{a}^{(1)}$ & $4.350 \mathrm{a}$ & 1,0 & 1,0 \\
\hline Corsum $\left(^{*}\right)$ & $(300+2100)$ & $147,8 \mathrm{a}$ & $4.288 \mathrm{a}$ & 1,0 & 1,0 \\
\hline Corsum $\left({ }^{*}\right)$ & $(360+2520)$ & $154,2 \mathrm{a}$ & $4.513 \mathrm{a}$ & 1,0 & 1,0 \\
\hline Dual & 1920 & $152,5 \mathrm{a}$ & $4.313 \mathrm{a}$ & 1,0 & 1,0 \\
\hline Dual & 2400 & $155,5 \mathrm{a}$ & $4.362 \mathrm{a}$ & 1,0 & 1,0 \\
\hline Dual & 2880 & $149,0 \mathrm{a}$ & $4.488 \mathrm{a}$ & 1,0 & 1,0 \\
\hline $\begin{array}{l}\text { Testemunha } \\
\text { capinada }\end{array}$ & --- & $149,8 \mathrm{a}$ & $4.575 \mathrm{a}$ & 1,0 & 1,0 \\
\hline $\begin{array}{l}\text { Testemunha } \\
\text { sem capina }\end{array}$ & $-\cdots$ & $23,5 b$ & $450 \mathrm{~b}$ & 1,0 & 1,0 \\
\hline Média Geral & & 138,4 & 4.072 & 1,0 & 1,0 \\
\hline Valor de F & & $82,76(* *)$ & $22,77(* *)$ & & \\
\hline DMS & & 20,7 & 1,245 & & \\
\hline C.V.(\%) & & 6,09 & 12,43 & & \\
\hline
\end{tabular}

(1) As médias na mesma coluna, seguidas pela mesma letra não diferem estatisticamente entre si, pelo teste de Tukey ao nivel de $5 \%$ de probabilidade.

(*) Mistura formulada.

(*) Significativo ao nivel de $1 \%$ de probabilidade.

DAT $=$ Dias após tratamento. 
Para o controle das plantas daninhas denominadas fazendeiro e capim-marmelada, todos os herbicidas nas doses estudadas apresentaram excelente indice, com $100 \%$ de controle, até 45 dias após a aplicação (Tabelas 4 e 5). Apenas a espécie leiteiro não apresentou resultado satisfatório, constatando-se indice nulo no controle.

TABELA 4 - PERCENTAGENS MÉDIAS DE CONTROLE DE PLANTA DANINHA DE MAIOR ABUNDÂNCIA, NA AVALIAÇÃO DA EFICÁCIA BIOLÓGICA DE HERBICIDAS APLICADOS NA PRÉEMERGÊNCIA, PARA O CONTROLE DE Galinsoga parviflora NA CULTURA DA BATATA - SETE LAGOAS (MG) - 1995

\begin{tabular}{|c|c|c|c|c|}
\hline \multirow[t]{2}{*}{ Tratamentos } & \multirow{2}{*}{$\begin{array}{c}\text { Doses } \\
\text { (g/ha i. a.) }\end{array}$} & \multicolumn{3}{|c|}{ Galinsoga parviflora (**) } \\
\hline & & $15 \mathrm{DAT}$ & $30 \mathrm{DAT}$ & 45 DAT \\
\hline Corsum $\left({ }^{*}\right)$ & $(240+1680)$ & 98 & 100 & 100 \\
\hline Corsum $(*)$ & $(300+2100)$ & 100 & 100 & 100 \\
\hline Corsum $(*)$ & $(360+2520)$ & 100 & 100 & 100 \\
\hline Dual & 1920 & 100 & 100 & 100 \\
\hline Dual & 2400 & 100 & 100 & 100 \\
\hline Dual & 2880 & 100 & 100 & 100 \\
\hline Testemunha capinada & $-\cdots$ & 100 & 100 & 100 \\
\hline Testemunha sem capina & $\cdots$ & 0 & 0 & 0 \\
\hline Número de individuos $/ \mathrm{m}^{2}$ & & 14,0 & 17,25 & 19,0 \\
\hline
\end{tabular}

DAT $=$ Dias após tratamento. i.a. $=$ Ingrediente Ativo. $(*)$ Mistura formulada

(“) Não foram necessárias análises da variância entre tratamentos.

TABELA 5 - PERCENTAGENS MÉdIAS DE CONTROLE DE PLANTA DANINHA DE MAIOR ABUNDÂNCIA, NA AVALIAÇÄO DA EFICÁCIA BIOLÓGICA DE HERBICIDAS APLICADOS NA PRÉEMERGÊNCIA, PARA O CONTROLE DE Brachiaria plantaginea NA CULTURA DA BATATA - SETE LAGOAS (MG) - 1995

\begin{tabular}{|c|c|c|c|c|}
\hline \multirow[t]{2}{*}{ Tratamentos } & \multirow{2}{*}{$\begin{array}{c}\text { Doses } \\
\text { (g/ha i. a.) }\end{array}$} & \multicolumn{3}{|c|}{ Brachiaria plantaginea (**) } \\
\hline & & $15 \mathrm{DAT}$ & $30 \mathrm{DAT}$ & $45 \mathrm{DAT}$ \\
\hline Corsum $(*)$ & $(240+1680)$ & 100 & 100 & 100 \\
\hline Corsum $(*)$ & $(300+2100)$ & 100 & 100 & 100 \\
\hline Corsum $(*)$ & $(30+2520)$ & 100 & 100 & 100 \\
\hline Dual & 1920 & 100 & 100 & 100 \\
\hline Dual & 2400 & 100 & 100 & 100 \\
\hline Dual & 2880 & 100 & 100 & 100 \\
\hline Testemunha capinada & $\cdots$ & 100 & 100 & 100 \\
\hline Testemunha sem capina & $\cdots$ & 0 & 0 & 0 \\
\hline Número de individuos $/ \mathrm{m}^{2}$ & & 8,5 & 11,25 & 14,75 \\
\hline
\end{tabular}

DAT = Dias após tratamento. i.a. = Ingrediente Ativo. (*) Mistura formulada

(*) Não foram necessárias análises da variância entre tratamentos. 


\title{
4 CONCLUSÃO
}

De acordo com os resultados obtidos no presente ensaio de campo pode-se concluir que:

- Corsum (Metolachlor + Metribuzin) e Metolachlor, nas doses estudadas, demostraram eficácia no controle de Galinsoga parviflora (fazendeiro) e
Brachiaria plantaginea (capim-marmelada);

- Corsum (Metolachlor + Metribuzin) e Metolachlor, nas doses estudadas, são altamente seletivos para a cultura da batata, na aplicação de pré-
emergência em área total.

\begin{abstract}
It was evaluated the performance of the herbicides Corsum (Metolachlor $840 \mathrm{~g} / \mathrm{L}+$ for weed $120 \mathrm{~g} / \mathrm{L}$ ), and Dual (Metolachlor $960 \mathrm{~g} / \mathrm{L}$ ) applied in the pre-emergence phase The treatments were 1920,2400 and $2880 \mathrm{~g} / \mathrm{ha}$; weed $240+1680 ; 300+2100$ and $360+2520 \mathrm{~g} / \mathrm{ha}$; Metolachlor both Corsum and Metolachlor for the and not-weeded controls. The results pointed out that the control of Galinsoga parviflor the tested doses, demonstrated $100 \%$ of effectiveness in application. The field competition and Brachiaria plantaginea, persisting for 45 days after potato. In the height of the plants 60 the weeds presented reduction in the production of and their diameters, differ plation) and in the number of tubers doses and the weeded control. These hot detected among the herbicides in the tested EWRC of 1.0 up to 15 days after These herbicides are highly selective, presenting a grade
\end{abstract}

\section{REFERÊNCIAS BIBLIOGRÁFICAS}

CAMARGO FILHO, W. P., SUEYOSHI, M. de L. S., CAMARGO, A. M. M. P. de, MAZZEI, A. R. Produção e mercado de batata no Brasil no periodo de 1971-90. Agricultura em São Paulo, São Paulo, v. 40, n. 1, p. 183-204, 1993.

\section{CARDOSO, M. R. O., FERREIRA, F. A., DESSIMONI, D. P. G. Manejo o tratos culturais do batatal. Inf. Agropec., Belo} Horizonte, v. 7, n. 76, p. 30-34, abr. 1981

DARIO, P. W., VEIGA, J. S., DARIO, G. J. A. Avaliação da eficiência dos herbicidas Sencor 700 GRDA (Metribuzin) e Sencor 480 (Metribuzin) no controle de plantas daninhas ocorrentes na v. 13 , n. 1, p. 79, maio, 1995.
verum). Hortic. Bras., Brasilia, 
DAKER, A. A água na agricultura. 3.ed. Rio de Janeiro : Freitas Bastos, 1970. v. 3 (Irrigação e drenagem).

EWRC. Report of the third and fourth Meeting of the European Weed Research Council Committee on Methods. Weed Res., v. 4, p. 88, 1964.

MASCARENHAS, M. H. T., CASTRO, A. R., MACEDO, A. A., RESENDE, L. M. A., CORREA, L. G., TÔRRES, P. A., DINIZ, R. $\mathrm{S}$. Cenário futuro da bataticultura em Minas Gerais. Hortic. Bras., Brasília, v. 13, n. 1, p. 93, maio, 1995.

PARRAGA M. S., TOZANI, R., PEREIRA, A. L., CORREA, E. H. F. S., FERNANDES, A. C. Efeito de herbicidas no cultivo da batata (Solanum tuberosum) formada de semente botânica. In: CONGRESSO BRASILEIRO DE HERBICIDAS E PLANTAS DANINHAS, 17., 1988, Piracicaba. Resumos... Piracicaba : Legis Summa, 1988. p. 296-297.

GOMES, F.P. Curso de estatística experimental. 5.ed. São Paulo : Nobel, 1973. 430 p.

SAGHIR, A. R., MARKOULLIS, G. Effect of weed competition and herbicides on yield and quality of potatoes. In: BRIT. WEED CONT. CONF., 12., 1974. Proceedings ...1974. p. 533-539.

SANTOS, C. A. L. dos, ROZANSKI, A. Emprego de herbicidas na cultura da batata. In: CONGRESSO BRASILEIRO DE HERBICIDAS E ERVAS DANINHAS, 13., 1980, IIhéus-Itabuna. Resumos... Ilhéus-Itabuna : CEPLAC/SBHED, 1980. p. 80.

SILVA, A. A. da, CARMO, C. A. S. do. Controle do capim-marmelada (Brachiaria plantaginea) na cultura da batata no Estado de Espirito Santo. In: CONGRESSO BRASILEIRO DE HERBICIDAS E ERVAS DANINHAS, 13., 1980, Ilhéus-Itabuna. Resumos... Ilhéus-Itabuna : CEPLAC/SBHED, 1980. p. 80.

SOSA, P. M., TOZANI, R., PEREIRA, A. L., CORREA, E. H., FERNANDES, A. C. Produção de batata (Solanum tuberosum L.) através de semente botânica: efeito de herbicidas aplicados em pré e pós-emergência. Hortic. Bras., v. 6, n. 1, p. 81, maio, 1988.

WOOLFE, J. A. The nutritional value of the components of the tuber. In: THE POTATO in the human diet. Cambridge, 1987. p. 19-57. 